\title{
Singular Pretransitional Behavior of the Electric Field-Dependent Part of the Thermodynamic Quantities of Strongly Polar Mesogenic Liquids in the Isotropic Phase
}

\author{
J. JADŻYN ${ }^{a}$, J.-L. DÉJARDIN ${ }^{b}$ AND G. CZEChOWSKI ${ }^{a}$ \\ ${ }^{a}$ Institute of Molecular Physics, Polish Academy of Sciences \\ M. Smoluchowskiego 17, 60-179 Poznań, Poland \\ ${ }^{b}$ Groupe de Physique Statistique et Moléculaire, MEPS, Université de Perpignan \\ 52 Avenue Paul Alduy, 66860 Perpignan Cedex, France
}

(Received April 13, 2007)

\begin{abstract}
A new interpretation of the pretransitional temperature behavior of the static dielectric permittivity and its derivative in terms of the basic thermodynamic quantities: the internal energy, the entropy, and the Helmholtz free energy is presented. It was shown that in the case of strongly polar mesogenic compounds (as the homologous series of alkylcyanobiphenyls) the electric field-induced increments of both the internal energy and the entropy exhibit a critical-like temperature dependence in the vicinity of transition from the isotropic liquid to the nematic phase. As a most important pretransitional effect, it is shown that at a temperature of about 10 degrees before the phase transition, an electric field applied to the isotropic liquids induces an increase in the entropy $(\Delta S>0)$ instead of its decrease, as observed far from the phase transition or for the non-mesogenic dipolar liquids.
\end{abstract}

PACS numbers: 64.70.Md, 65.40.Gr, 77.22.Ch

\section{Introduction}

Phase transitions from the isotropic (I) liquid to the nematic (N) liquid crystal or between different liquid crystalline phases are ones of the most spectacular transitions in nature. These transitions are either weakly discontinuous or continuous, and, as such, they are classified as a weakly first order or close to the second order. At the N-I transition, one detects the enthalpy changes of the order 
of $1 \mathrm{~kJ} / \mathrm{mol}$, and often much less. At a typical first-order transition, namely in the melting from the crystalline solid $(\mathrm{Cr})$ to the isotropic liquid (or to the liquid crystalline phase), the enthalpy change is around 20-50 kJ/mol. Similarly, the molar volume jump associated to N-I transition typically is only a few percent of that observed at a melting process $[1,2]$. The thermodynamic weakness of the nematic to isotropic phase transition, which, as a matter of fact, is a consequence of a loss of the molecular orientational order at $T_{\mathrm{NI}}$, leads in turn to large pretransitional effects. In the prenematic region of the isotropic liquids one observes an abrupt increase not only in thermodynamic quantities, such as the specific heat, the thermal expansion, and the isothermal compressibility [2] but also in other physical properties: the static magnetic [3] and electric [4] birefringence, the light scattering [5], the nonlinear dielectric effect [6], the anomalously slow rotational diffusion [7-9], as well as the molecular spin-lattice relaxation [10, 11], the dynamic light scattering [12], the transient grating optical Kerr effect [13, 14], and the nonlinear dielectric relaxation $[15,16]$.

The present paper concerns the prenematic effects seen in the basic thermodynamic functions (internal energy, entropy, and the Helmholtz free energy), resulting from the temperature behavior of the static dielectric permittivity (or its derivative) of a number of compounds belonging to a series of alkylcyanobiphenyls.

\section{Experimental}

The compounds studied belong to the homologous series of the strongly polar (molecular dipole moment $\mu \approx 5 \mathrm{D}$ ) alkylcyanobiphenyls $\left(\mathrm{C}_{n} \mathrm{H}_{2 n+1} \mathrm{PhPhCN}\right.$, $n \mathrm{CB})$. The temperatures of the phase transitions studied are depicted in Fig. 1. For

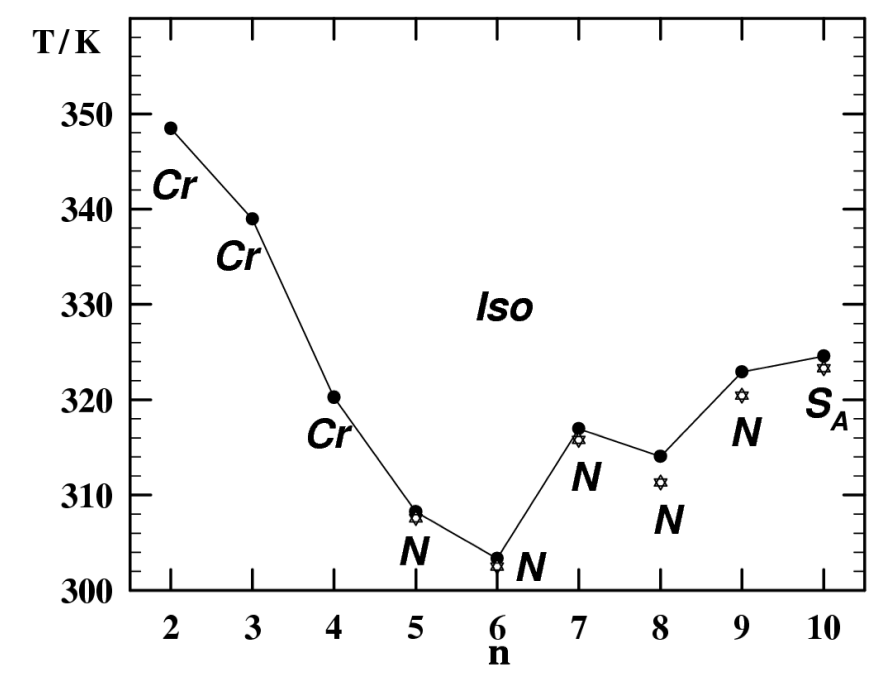

Fig. 1. Temperatures of the phase transitions of the homologous series of alkylcyanobiphenyls ( $n \mathrm{CB}, n=2-10$ ) studied. The stars denote the temperature of the virtual second-order transitions. 
$n=2-4$, the compounds are non-mesogenic, for $n=5-9$ the temperature decrease in the isotropic liquids leads to an appearance of the nematic phase and finally for $n=10$ the transition to the smectic $\mathrm{A}\left(\mathrm{S}_{\mathrm{A}}\right)$ phase occurs. The compounds were synthesized and purified at the Institute of Chemistry, Military University of Technology, Warsaw. The purity of the compounds, checked by chromatography, was better than $99.5 \%$. The measurements of the static dielectric properties of liquid $n$ CB's were performed with a HP 4194A impedance/gain phase analyzer in the kilohertz frequency range. The accuracy of the permittivity determination was better than $0.5 \%$. The measuring capacitor consisted of three plane electrodes: one central "hot" electrode and two grounded electrodes on each side. The electrical heating of high performance, with the use of UNIPAN $650 \mathrm{H}$ controller, assured a temperature stabilization better than $10^{-3} \mathrm{~K}$ and a possibility of the temperature change of $10^{-2} \mathrm{~K}$.

\section{Results and discussion}

Figure 2 presents the temperature dependences of the static dielectric permittivity $\left(\varepsilon_{\mathrm{S}}\right)$ measured for the isotropic $n \mathrm{CB}$ compounds in the vicinity of the phase transitions showed in Fig. 1. A quite well-marked difference in the $\varepsilon_{\mathbf{S}}(T)$ dependence, observed in the vicinity of the first-order ( $\mathrm{Cr}-\mathrm{I}$ transition for $n=2-4$ ) and the weakly first-order $\left(\mathrm{N}-\mathrm{I}\right.$ or $\mathrm{N}-\mathrm{S}_{\mathrm{A}}$ for $\left.n=5-10\right)$ phase transitions, reflects a difference in the temperature behavior of the basic thermodynamic quantities due to the application of an external electric field to the system. As firstly shown by Fröhlich in 1958 [17] and next by Becker [18], Landau et al. [19] and Scaife [20], the density of the basic thermodynamic quantities, namely the internal energy $U$,

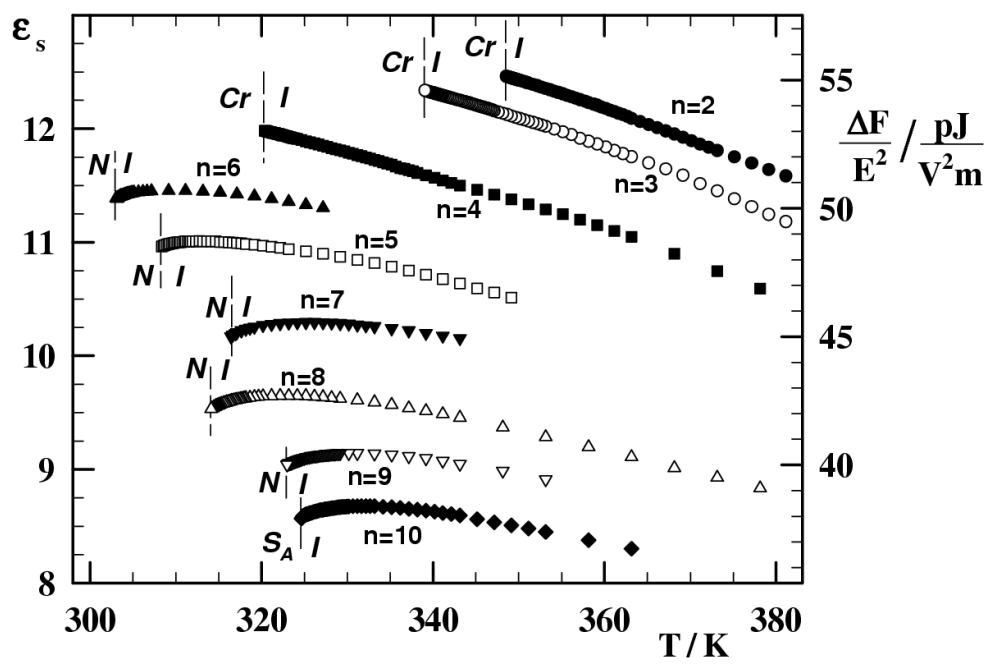

Fig. 2. Temperature dependences of the static dielectric permittivity measured in the isotropic phase of $n \mathrm{CB}$ series $(n=2-10)$. 
the entropy $S$, and the Helmholtz free energy $F$ are related to the static permittivity $\varepsilon_{\mathrm{s}}$ or its derivative $\mathrm{d} \varepsilon_{\mathrm{s}} / \mathrm{d} T$ and the applied electric field strength $E$ as follows:

$$
\begin{aligned}
& U(T, E)=U_{0}(T)+\frac{\varepsilon_{0}}{2}\left(\varepsilon_{\mathrm{s}}+T \frac{\partial \varepsilon_{\mathrm{s}}}{\partial T}\right) E^{2}, \\
& S(T, E)=S_{0}(T)+\frac{\varepsilon_{0}}{2} \frac{\partial \varepsilon_{\mathrm{s}}}{\partial T} E^{2}, \\
& F(T, E)=F_{0}(T)+\frac{\varepsilon_{0}}{2} \varepsilon_{\mathrm{s}} E^{2},
\end{aligned}
$$

where $U_{0}, S_{0}$, and $F_{0}$ denote the values of the thermodynamic quantities in the absence of the electric field, $T$ is the absolute temperature and $\varepsilon_{0}=8.85 \mathrm{pF} / \mathrm{m}$ is the permittivity of free space.

In Eq. (1), the term $0.5 \varepsilon_{0} T \partial \varepsilon_{\mathrm{s}} / \partial T$ represents a part of the dielectric internal energy per unit electric field strength and per unit volume, which is converted into heat during the isothermal charging of the capacitor filled with a dielectric material having the static permittivity $\varepsilon_{\mathrm{s}}$. The heat is drawn away from the capacitor by its surrounding. The remaining term is the electric free energy (Eq. (3)) stored in the capacitor and the difference $\Delta F=F(T, E)-F_{0}(T)$ represents a maximum of the energy which can be yielded during the isothermal discharge of the capacitor. Equation (3) shows that the temperature behavior of the electric part of the free energy density per unit $E$ behaves as the static permittivity itself, as illustrated in Fig. 2. A relatively small drop of the free energy, observed for mesogenic compounds with decreasing temperature, announces an approaching of the transition to the liquid crystalline phase. Both the internal energy and the entropy, given by Eqs. (1) and (2), respectively, depend on the permittivity derivative.

Figures 3 and 4 present the temperature dependences of the field-induced increments of the internal energy and the entropy, respectively. The corresponding data for the non-mesogenic compound of $n=4$ (the polarity of which is the same as that of all the other compounds studied) are presented in the figures, for comparison. Both $\Delta U / E^{2}$ and $\Delta S / E^{2}$ exhibit a critical-like temperature behavior, which can be described by the following equation [21]:

$$
\frac{\Delta X}{E^{2}}=A_{x}+B_{x}\left(T-T_{x}^{*}\right)+C_{x}\left(T-T_{x}^{*}\right)^{-\alpha_{x}},
$$

where $X$ stands for $U$ or $S$. The first two terms of the above equation describe a linear background and the values of the constants $A$ and $B$ are determined from the corresponding dependences obtained for the non-mesogenic compound of $n=4$ (straight lines in Figs. 3 and 4). $T^{*}$ is the extrapolated temperature of a virtual continuous phase transition, $C$ is a constant and $\alpha$ is a critical exponent: they are three fitting parameters for describing $\Delta U / E^{2}$ and $\Delta S / E^{2}$ experimental data by Eq. (4).

For a given compound, the best fits of Eq. (4) to both the internal energy and the entropy temperature dependences gave practically the same value of $T^{*}$ (the differences do not exceed $\pm 0.1 \mathrm{~K}$ ), denoted as stars in Fig. 1 . The differences 


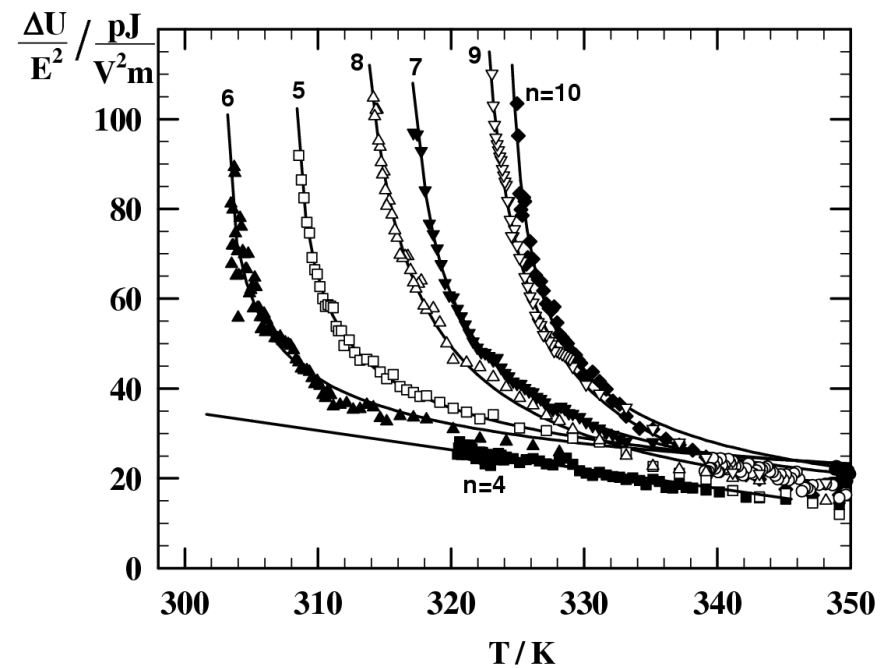

Fig. 3. Critical-like temperature dependence of the electric field-induced increment of the internal energy density of mesogenic $n$ CB's. The solid lines represent the best fits of Eq. (1) to the experimental data.

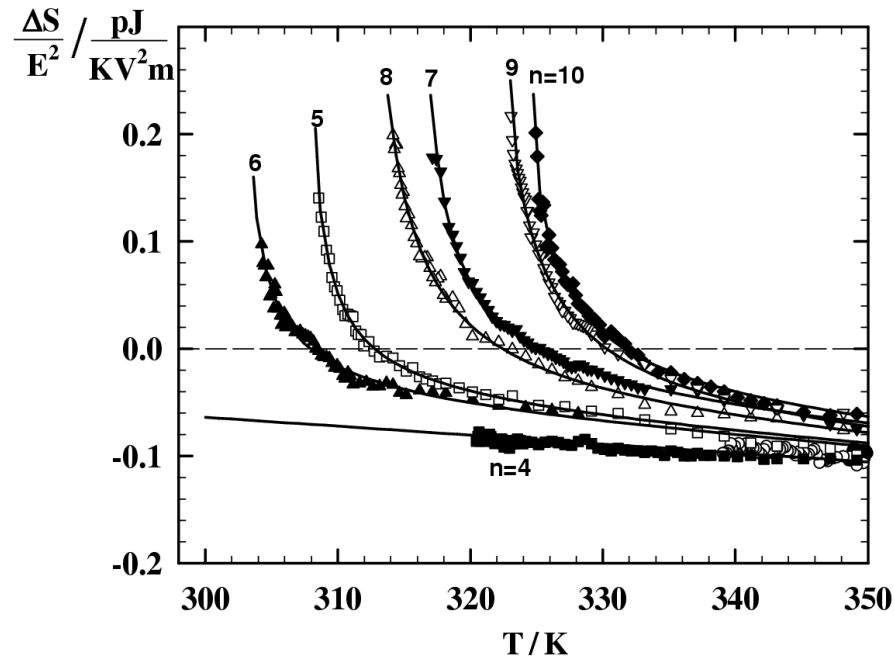

Fig. 4. Critical-like temperature dependence of the electric field-induced increment of the entropy density of mesogenic $n$ CB's. The solid lines represent the best fits of Eq. (2) to the experimental data.

$\Delta T=T_{\mathrm{NI}}-T^{*}$ obtained for most of the compounds studied are less than $1 \mathrm{~K}$, with an exception for $8 \mathrm{CB}$ and $9 \mathrm{CB}$, where $\Delta T$ is about $1.5 \mathrm{~K}$. The values of $\Delta T$ obtained are characteristic of a rather weakly discontinuous character of the $\mathrm{N}-\mathrm{I}$ transition in cyanobiphenyl compounds. The values of the critical exponents 
are following: $\alpha_{u}=0.8 \pm 0.1$ (internal energy) and $\alpha_{s}=0.5 \pm 0.2$ (entropy) for all the compounds studied. The latter value agrees well with those obtained in previous papers [21-23], where the temperature dependence of the permittivity and its derivative were analyzed and interpreted within the tricritical hypothesis of the N-I transition [24].

A special attention must be drawn with respect to the entropy, where the sign of the field-induced increment $\Delta S=S(T, E)-S_{0}(T)$ depends straightly on the sign of the permittivity derivative. A negative value of $\mathrm{d} \varepsilon_{\mathrm{s}} / \mathrm{d} T$, i.e. $\Delta S<0$, means that the entropy diminishes due to an electric field applied to the dielectric material, indicating an increase in the molecular order. It is a normal behavior of dipolar liquids for which the static permittivity increases when the temperature decreases. However, in the case of the mesogenic compounds studied here the situation is more complicated. As shown in Fig. 4, at a temperature of somewhat less than 10 degrees before the $\mathrm{I}-\mathrm{N}$ transition of a given $n \mathrm{CB}$ compound, the increment of the entropy changes its sign and becomes positive, $\Delta S>0$, therefore, an electric field applied to the isotropic dipolar liquid causes a decrease in the order on the molecular level. A field-induced increase in the entropy is possible only when the molecules are (at least partially) self-organized in the isotropic liquid with a predominance of the antiparallel dipolar arrangement within the intermolecular entities formed.

The singular entropy effect observed is a result of a competition between the spontaneous antiparallel dipolar self-assembling in the form of the prenematic domains and the parallel dipolar ordering forced by the electric field. As the temperature approaches to the transition to the nematic phase, the effect is strongly enhanced and its behavior takes a critical-like form which is certainly connected with a strong increase in the prenematic domains size, i.e., with an increase in the number of the self-organized molecules. In such a case of heterogeneity of isotropic liquid, even if it exists on the microscopic level only, one can expect two orientational effects caused by an applied electric field: the first effect is a normal orientation of "free" dipolar molecules (a very great majority) and the second one is an orientation of molecules assembled and antiparallel coupled in the prenematic domains. Consequently, two relaxation processes can be expected in such systems.

The relaxation due to a return of the molecular dipoles to their random distribution in the liquid state, after the perturbation caused by an external electric field, is quite easily detectable with the use of the conventional dielectric spectroscopy. The detection of the relaxation process connected with reorientational transformations forced by an external electric field within the prenematic domains is, from the experimental point of view, an extremely difficult task mainly due to a relatively small amount of the molecules engaged in the domains (the liquid is transparent even if observed with the optical microscope). Only when an electric field of a very high strength is applied to the mesogenic liquid, causing the nonlinearity of its dielectric polarization vs. the electric field strength, then 
a new dielectric absorption band appears at the frequency of about one order of magnitude lower than that of "normal" dielectric relaxation. The details on the nonlinear dielectric relaxation experiments are described in papers [15, 16].

Finally, it seems important to mention here that a general assertion, often used in papers on the isotropic liquids, stating that an increase in the dielectric permittivity with decreasing temperature is a direct result of an increase in the dipoles density, does not always holds. The data presented in this paper show that in the case of a molecular self-organization, like in the pretransitional region of isotropic mesogenic compounds, where the pseudonematic domains are formed, a decrease in the temperature followed by an increase in the dipoles density, can lead to the reverse effect, i.e. to the diminishing of the dielectric permittivity. The deciding factor is here a subtle competition between the dipole-dipole and dipole-electric field interactions taking place in molecular liquids.

\section{References}

[1] P.G. de Gennes, Physics of Liquid Crystals, Oxford University Press, Oxford 1974.

[2] S. Singh, Phys. Rep. 324, 107 (2000).

[3] J.D. Litster, T.W. Stinson III, J. Appl. Phys. 41, 996 (1970).

[4] J.C. Filippini, Y. Poggi, Phys. Lett. A 65, 30 (1978).

[5] T.D. Gierke, W.H. Flygare, J. Chem. Phys. 61, 2231 (1974).

[6] A. Drozd-Rzoska, S.J. Rzoska, J. Zioło, K. Czupryński, J. Phys., Condens. Matter 43, L473 (1999).

[7] J. Jadżyn, J.-L. Déjardin, G. Czechowski, M. Ginovska, J. Phys., Condens. Matter $\mathbf{1 7}, 813(2005)$.

[8] J.-L. Déjardin, J. Jadżyn, J. Chem. Phys. 123, 174502 (2005).

[9] J. Jadżyn, D. Bauman, J.-L. Déjardin, M. Ginovska, G. Czechowski, Acta Phys. Pol. A 108, 479 (2005).

[10] B. Cabane, G. Clarke, Phys. Rev. Lett. 25, 91 (1970).

[11] S. Gosh, E. Tettamanti, E. Indovina, Phys. Rev. Lett. 29, 638 (1973).

[12] T.W. Stinson III, J.D. Litster, Phys. Rev. Lett. 25, 503 (1970).

[13] F.W. Deeg, S.R. Greenfield, J.J. Stankus, V.J. Newell, M.D. Fayer, J. Chem. Phys. 93, 3503 (1990).

[14] J.J. Stankus, R. Torre, C.D. Marshall, S.R. Greenfield, A. Sengupta, A. Tokmakoff, M.D. Fayer, Chem. Phys. Lett. 194, 213 (1992).

[15] P. Kȩdziora, J. Jadżyn, L. Hellemans, Phys. Rev. E 66, 021709,031702 (2002).

[16] J. Jadżyn, P. Kędziora, L. Hellemans, in: Relaxation Phenomena in Dielectric, Magnetic and Superconducting Materials, Eds. W. Haase, S. Wróbel, Springer-Verlag, Heidelberg 2003, p. 51.

[17] H. Fröhlich, Theory of Dielectrics, 2nd ed., Clarendon Press, Oxford 1958.

[18] R. Becker, Electromagnetic Fields and Interactions, Ed. F. Sauter, Blackie and Son, London 1964. 
[19] L.D. Landau, E.M. Lifshitz, L.P. Pitaevskii, Electrodynamics of Continuous Media, 2nd ed., Pergamon Press, Oxford 1984.

[20] B.K.P. Scaife, Principles of Dielectrics, Clarendon Press, Oxford 1998.

[21] J. Rzoska, J. Zioło, W. Sułkowski, J. Jadżyn, G. Czechowski, Phys. Rev. E 64, 052701 (2001).

[22] J. Jadżyn, G. Czechowski, C. Legrand, R. Douali, Phys. Rev. E 67, 041705 (2003).

[23] M. Ginovska, H. Kresse, D. Bauman, G. Czechowski, J. Jadżyn, Phys. Rev. E 69, 022701 (2004).

[24] A. Żywocinski, J. Phys. Chem. B 107, 9491 (2003). 